\title{
Analytical Modeling of Soil Solution Monitoring by Diffusion in Porous Cups
}

\author{
Benjamin D. Shaw • Atac Tuli · Jing-Bin Wei • \\ Jan W. Hopmans
}

Received: 3 June 2008 / Accepted: 15 April 2009 / Published online: 7 May 2009

(C) The Author(s) 2009. This article is published with open access at Springerlink.com

\begin{abstract}
There is increasing interest toward in situ solution monitoring of soil chemicals for agricultural, industrial, and ecological purposes. Rather than extracting soil solution, a series of laboratory experiments was conducted to evaluate a diffusion equilibration technique, providing for real time in situ soil solution nitrate concentration via UV absorption spectroscopy. Experiments allowed for diffusion of nitrate from an outside reservoir into a porous cup. The experimental data were compared with model predictions using three different analytical solutions: (i) an exact solution (EX model) assuming radial symmetry that includes a porous cup with a porous wall and a finite size outside reservoir, (ii) an approximate solution ( $\mathrm{RC}$ model) that assumes an infinitely large supply reservoir with a porous cup wall that does not influence ion diffusion, and (iii) and a model based on an electric circuit analog (ECA model). The results indicate that the ECA solution provides the best match overall to the experimental data.
\end{abstract}

Keywords Nitrate diffusion - Soil solution - Exact analytical solution · Electrical circuit analog solution · UV absorption spectrometer · Stainless steel porous cup

\section{Introduction}

There is increasing interest toward in situ solution monitoring of soil chemicals for agricultural, industrial, and ecological purposes. Yet, soil solution sampling is complicated as conventional methods require soil water extraction by suction with subsequent analysis of extracted samples in the laboratory. Weihermuller et al. (2007) presented a comprehensive review of readily used in situ soil water extraction methods to monitor soil solute concentration

B. D. Shaw · J.-B. Wei

Department of Mechanical and Aeronautical Engineering, UC Davis, Davis, CA 95616, USA

A. Tuli $(\varangle) \cdot$ J. W. Hopmans

Department of Land, Air and Water Resources, UC Davis, Davis, CA 95616, USA

e-mail: atuli@ucdavis.edu 
changes as brought about by solute transport processes. They presented various limitations of each of the soil solution extraction methods.

An alternative approach to monitoring soil solution concentration is by applying the passive diffusion technique, introduced by Moutonnet et al. (1993), allowing for ionic equilibrium between the solution inside the porous cup and the surrounding soil solution by ionic diffusion. Whereas, the diffusion method is simple and allows simultaneous measurement of soil water retention, equilibration times are likely to be at time scales of days and longer. Riga and Charpentier (1998) developed a mathematical method to estimate equilibrium time and they showed that equilibration times for nitrate diffusion can be weeks, especially for unsaturated soils. A field evaluation of both the diffusion and vacuum extraction methods was conducted by Poss et al. (1995). They concluded that both sampling devices are suitable for nitrate leaching monitoring, considering that the diffusion method provides for a much more time-integrated measurement, whereas soil solute concentration determined with the extraction method reflects the concentration at the time of the solute extraction.

Diffusion experiments based on the equilibration concepts were conducted for a range of experimental conditions by Tuli et al. (2009). The main objective of the present study was to develop and compare analytical solutions of the ionic diffusion method, using laboratory experiments of nitrate diffusion in stainless steel (SS) porous cups. The experiments considered diffusion in aqueous systems, i.e., diffusion in soil was not considered. While, it is worthwhile, to perform measurements with soil, for validation of the mathematical solutions, and the experimental methods, we focused on completely aqueous systems in the present studies. The extension to measurements in soils will be performed in future studies.

\section{Experimental Methods}

\subsection{Diffusion Experiments}

In order to test the diffusion equilibration method, we conducted a series of nitrate diffusion experiments into a stainless steel (SS) porous cup filled with de-ionized water by, way of an UV absorption method, using fiber-optic technology. Analytical solutions to ion diffusion were fitted to diffusion data, providing for solute diffusion coefficients for solution into the porous SS cup. The experiments were conducted for liquid diffusion across a range of outside reservoir volumes. The detailed experimental procedure including a schematic of the UV dip probe was presented in Tuli et al. (2009).

The simple experimental set up consists of a SS porous cup with inside radius of $r_{1}$ containing a known volume of de-ionized water, where $C_{I}(r, 0)=0$, and an outside reservoir of inside radius $r_{3}(\mathrm{~L})$, containing a known volume of $\mathrm{NO}_{3}{ }^{-}-\mathrm{N}$ solution at predetermined concentration (Fig. 1). We denote the outside radius of the SS cup as $r_{2}$ (L), to include the thickness of the SS cup wall, $w_{t}$. At $\mathrm{t}=0$, the stainless steel cup filled with de-ionized water was immersed into the reservoir filled with the nitrate solution. The optical probe and the inner and outer cups were all positioned concentrically, i.e., their centerlines were aligned.

In order to investigate the size effect of the reservoir cup to nitrate diffusion, experiments with a range of ratio values, as defined by the outside radius $\left(r_{2}\right)$ of a SS cup divided by radius of outside reservoir $\left(r_{3}\right)$, or $z_{2}=r_{2} / r_{3}$. This set of experiments was chosen to establish a range of diffusion equilibration times, which are influenced by the size of the outer reservoir, for the SS Cup with a porosity of $0.151 \mathrm{~m}^{3} \mathrm{~m}^{-3}$ (Table 1). The values for $z_{2}$ were 0.16 , $0.31,0.50$, and 0.82 . This range in $z_{2}$ values was obtained by employing different outside reservoirs with different diameters (Fig. 1) where the inside cup was always the same. For 


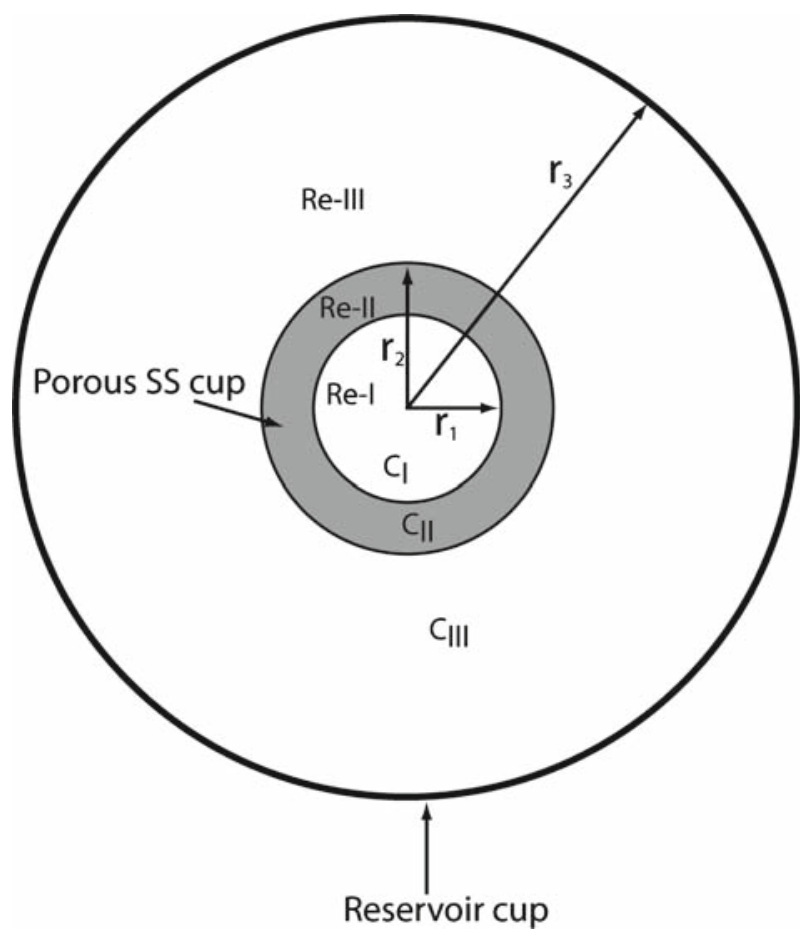

Fig. 1 Schematic of the experimental system with regions I (inside porous cup), II (cup wall), and III (outside supply reservoir)

each of the four experiments, we filled the outside reservoir with a predetermined volume $\left(V_{\text {III }}\right.$ ) of $50 \mathrm{mg} \mathrm{l}^{-1} \mathrm{NO}_{3}^{-}-\mathrm{N}$ solution (using $\mathrm{KNO}_{3}$ ) for which the ionic diffusion coefficient in water $\left(D_{\mathrm{o}}\right)$ is $1.902 \times 10^{-5} \mathrm{~cm}^{2} \mathrm{~s}^{-1}$ (Vanysek 2008). Subsequently, the SS cup was filled with de-ionized water and placed in the center of the outside reservoir at $t=0$. For $t>0$, the concentration changes inside the SS cup as caused by diffusion were monitored continuously using the UV dip probe (Tuli et al. 2009). The reservoir was covered with parafilm to prevent evaporation during the diffusion experiments. Diffusion experiments were conducted until the concentration in stainless steel cup was about $90 \%$ of the final $\mathrm{NO}_{3}{ }^{-}-\mathrm{N}$ equilibrium concentration (Riga and Charpentier 1998; Tuli et al. 2009). We do not expect that thermal convection was a factor in these experiments. When the experiments were performed, the liquids would have been thermally equilibrated considering the long time scales associated with species diffusion. The experimental parameters are given in Table 2 .

Representative experimental results on measured concentration versus time are shown in Fig. 2 for different values of $z_{2}$. This figure also shows plots obtained from the application of various models. The experimental results and their comparisons with the analytical models will be discussed in detail later in this article.

\subsection{Ultraviolet Dip Probe}

The fiber-optic T300-RT-UV-VIS transmission dip probe $(6.35 \mathrm{~mm}$ diameter; Ocean Optics, Inc., Dunedin, FL) uses a two-channel spectrometer and light source to measure absorbance and transmission of the soil solution. By way of this technique, light is transmitted from the 
Table 1 Properties of the stainless steel (SS) porous cup

\begin{tabular}{ll}
\hline Property & SS Cup \\
\hline Media grade $($ Mott Corp) $(\mu \mathrm{m})$ & 0.2 \\
Air entry value $(\mathrm{cm})$ & 240 \\
Outside diameter $(\mathrm{cm})$ & 1.908 \\
Inside diameter $(\mathrm{cm})$ & 1.572 \\
Outside height $(\mathrm{cm})$ & 3.864 \\
Inside height $(\mathrm{cm})$ & 3.758 \\
Wall thickness $(\mathrm{cm})$ & 0.168 \\
Porosity $(\phi),\left(\mathrm{cm}^{3} \mathrm{~cm}^{-3}\right)$ & 0.151 \\
Hydraulic conductivity $\left(K_{\mathrm{S}}\right),\left(\mathrm{cm} \mathrm{h}^{-1}\right)$ & $1.095 \times 10^{-2}$ \\
Permeability $(\mathrm{k})(\mathrm{cm})^{2}$ & $2.432 \times 10^{-11}$ \\
\hline
\end{tabular}

Table 2 Parameters used in estimation of the diffusion coefficient for each $z_{2}$ case

\begin{tabular}{|c|c|c|c|c|c|c|c|c|c|c|c|}
\hline \multirow[t]{2}{*}{ Experiment } & \multirow[t]{2}{*}{$z_{2}$} & $r_{2}$ & $r_{1}$ & $w_{\mathrm{t}}$ & $r_{3}$ & 1 & $V_{\mathrm{I}}$ & $V_{\mathrm{III}}$ & $V_{\mathrm{t}}$ & $C_{2}$ & $\bar{C}_{\text {ave }}$ \\
\hline & & \multicolumn{5}{|l|}{$\mathrm{cm}$} & \multicolumn{3}{|l|}{$\mathrm{ml}$} & \multicolumn{2}{|c|}{$\mathrm{mg}^{-1}$} \\
\hline 1 & 0.16 & 0.95 & 0.79 & 0.17 & 6.03 & 3.86 & 5.5 & 363.5 & 369.0 & 50 & 49.3 \\
\hline 2 & 0.31 & 0.95 & 0.79 & 0.17 & 3.03 & 3.86 & 5.9 & 100.5 & 106.4 & 50 & 47.2 \\
\hline 3 & 0.50 & 0.95 & 0.79 & 0.17 & 1.93 & 3.86 & 5.1 & 29.7 & 34.8 & 50 & 42.7 \\
\hline 4 & 0.82 & 0.95 & 0.79 & 0.17 & 1.17 & 3.86 & 5.2 & 5.2 & 10.3 & 50 & 24.9 \\
\hline
\end{tabular}

illumination fiber through a plano-convex lens and through the sample compartment to a flat, second-surface mirror that is placed $5 \mathrm{~mm}$ away from the lens. The reflected light is focused by the lens onto the read fiber, which transfers the attenuated light back to the spectrometer across a total path length of $10 \mathrm{~mm}$. (Fig. 1 in Tuli et al. 2009). In principle, when light passes through the $\mathrm{NO}_{3}{ }^{-}-\mathrm{N}$ solution, the light interacts with the $\mathrm{NO}_{3}{ }^{-}-\mathrm{N}$ through scattering and absorption, with attenuation peaks at wavelengths of about 201 and $302 \mathrm{~nm}$. In order to obtain useful absorption data, a wavelength range between 235 and $240 \mathrm{~nm}$ was selected. In this way, the absorbance spectra can provide accurate results, while still allowing strong (but not saturated) signals with minimum detectable concentration values of about $1 \mathrm{mgl}^{-1}$ (Tuli et al. 2009).

\section{Types of Models}

\subsection{Governing Equations for Exact Analytical Solution}

We consider the situation where species transport is only from diffusion and occurs only in the radial direction. We will solve the initial-value problem where a porous cup filed with fluid that has a diffusive species that is suddenly exposed to fluid on its outside, where the cup inner radius is $r_{1}$, the cup outer radius is $r_{2}$, and the inner radius of the container holding the outer fluid is $r_{3}$ (Fig. 1). There are three regions where the following diffusion equations need to be solved (Fig. 1). 


$$
\begin{gathered}
\text { Region I (Re-I) : } \frac{\partial C_{\mathrm{I}}}{\partial t}=D_{\mathrm{o}}\left(\frac{\partial^{2} C_{\mathrm{I}}}{\partial r^{2}}+\frac{1}{r} \frac{\partial C_{\mathrm{I}}}{\partial r}\right), \quad 0<r<r_{1} \\
\text { Region II (Re-II) }: \phi \frac{\partial C_{\mathrm{II}}}{\partial t}=D_{\mathrm{w}}\left(\frac{\partial^{2} C_{\mathrm{II}}}{\partial r^{2}}+\frac{1}{r} \frac{\partial C_{\mathrm{II}}}{\partial r}\right), \quad r_{1}<r<r_{2} \\
\text { Region III (Re-III) }: \frac{\partial C_{\mathrm{III}}}{\partial t}=D_{\mathrm{o}}\left(\frac{\partial^{2} C_{\mathrm{III}}}{\partial r^{2}}+\frac{1}{r} \frac{\partial C_{\mathrm{III}}}{\partial r}\right), \quad r_{2}<r<r_{3}
\end{gathered}
$$

The variable $C$ denotes concentration, $D_{\mathrm{o}}$ is the molecular species diffusivity in aqueous solution, $D_{\mathrm{w}}$ is the effective species diffusivity of the porous cup, and $\phi$ is the cup porosity. We assume that species transport is only due to radial diffusion.

These equations are to be solved subject to the following boundary conditions (applied at $r=0$ and $r=r_{3}$ ) and interface conditions (i.e., internal boundary conditions applied at $r=r_{1}$ and $r=r_{2}$ ).

$$
\begin{gathered}
\left.\frac{\partial C_{\mathrm{I}}}{\partial r}\right|_{r=0}=0,\left.\quad \frac{\partial C_{\mathrm{III}}}{\partial r}\right|_{r=r_{3}}=0 \\
\left.C_{\mathrm{I}}\right|_{r=r_{1}}=\left.C_{\mathrm{II}}\right|_{r=r_{1}},\left.\quad C_{\mathrm{II}}\right|_{r=r_{2}}=\left.C_{\mathrm{III}}\right|_{r=r_{2}} \\
\left.D_{\mathrm{O}} \frac{\partial C_{\mathrm{I}}}{\partial r}\right|_{r=r_{1}}=\left.D_{\mathrm{W}} \frac{\partial C_{\mathrm{II}}}{\partial r}\right|_{r=r_{1}},\left.D_{\mathrm{W}} \frac{\partial C_{\mathrm{II}}}{\partial r}\right|_{r=r_{2}}=\left.D_{\mathrm{o}} \frac{\partial C_{\mathrm{III}}}{\partial r}\right|_{r=r_{2}}
\end{gathered}
$$

The initial condition is as follows:

$$
C_{\mathrm{I}}(r, 0)=C_{1}, \quad C_{\mathrm{II}}(r, 0)=C_{1}, \quad C_{\mathrm{III}}(r, 0)=C_{2} .
$$

We will focus on obtaining analytical rather than numerical solutions of the governing equations. Numerical methods can be useful (or even the only possible solution method) in some circumstances, but numerical methods often do not yield the physical interpretations that an analytical solution provides, e.g., in terms of determining which parameters, or combinations of parameters, are dominant. Another advantage to having an analytical solution is that it can provide a baseline solution that a numerical solution can be compared with, thus allowing the accuracy of the numerical solution to be evaluated.

\subsection{Exact Solution}

We non-dimensionalize the equations by defining the variables $\xi_{i}=\left(C_{i}-C_{2}\right) /\left(C_{1}-C_{2}\right), i=$ I, II, and III; $z=r / r_{3}, z_{1}=r_{1} / r_{3}, z_{2}=r_{2} / r_{3}, \tau=D_{\mathrm{o}} t / r_{3}^{2}$, and $\gamma=D_{\mathrm{w}} / D_{\mathrm{o}}$, which leads to the following equations:

$$
\begin{aligned}
& \frac{\partial \xi_{\mathrm{I}}}{\partial \tau}=\left(\frac{\partial^{2} \xi_{\mathrm{I}}}{\partial z^{2}}+\frac{1}{z} \frac{\partial \xi_{\mathrm{I}}}{\partial z}\right), \quad 0<z<z_{1} \\
& \frac{\partial \xi_{\mathrm{II}}}{\partial \tau}=\frac{\gamma}{\phi}\left(\frac{\partial^{2} \xi_{\mathrm{II}}}{\partial z^{2}}+\frac{1}{z} \frac{\partial \xi_{\mathrm{II}}}{\partial z}\right), \quad z_{1}<z<z_{2} \\
& \frac{\partial \xi_{\mathrm{III}}}{\partial \tau}=\left(\frac{\partial^{2} \xi_{\mathrm{III}}}{\partial z^{2}}+\frac{1}{z} \frac{\partial \xi_{\mathrm{III}}}{\partial z}\right), \quad z_{2}<z<1 \\
& \left.\frac{\partial \xi_{\mathrm{I}}}{\partial z}\right|_{\mathrm{z}=0}=0,\left.\quad \frac{\partial \xi_{\mathrm{III}}}{\partial z}\right|_{\mathrm{z}=1}=0 \\
& \left.\xi_{\mathrm{I}}\right|_{\mathrm{r}=\mathrm{r}_{1}}=\left.\xi_{\mathrm{II}}\right|_{\mathrm{r}=\mathrm{r}_{1}},\left.\quad \xi_{\mathrm{II}}\right|_{\mathrm{r}=\mathrm{r}_{2}}=\left.\xi_{\mathrm{III}}\right|_{\mathrm{r}=\mathrm{r}_{2}}
\end{aligned}
$$




$$
\begin{aligned}
& \left.\frac{\partial \xi_{\mathrm{I}}}{\partial z}\right|_{\mathrm{z}=\mathrm{z}_{1}}=\left.\gamma \frac{\partial \xi_{\mathrm{II}}}{\partial z}\right|_{\mathrm{z}=\mathrm{z}_{1}},\left.\quad \gamma \frac{\partial \xi_{\mathrm{II}}}{\partial z}\right|_{\mathrm{z}=\mathrm{z}_{2}}=\left.\frac{\partial \xi_{\mathrm{III}}}{\partial z}\right|_{\mathrm{z}=\mathrm{z}_{2}} \\
& \xi_{\mathrm{I}}(z, 0)=1, \quad \xi_{\mathrm{II}}(z, 0)=1, \quad \xi_{\mathrm{III}}(z, 0)=0 .
\end{aligned}
$$

The exact solution, to these equations, is developed by applying the method of separation of variables in each region and then applying the interface and boundary conditions (Tittle 1965; de Monte 2000, 2002). In order to this end, we assume the following product solutions:

$$
\begin{gathered}
\xi_{\mathrm{I}}=h_{\mathrm{I}}(z) g_{\mathrm{I}}(\tau), \quad 0<z<z_{1} \\
\xi_{\mathrm{II}}=h_{\mathrm{II}}(z) g_{\mathrm{II}}(\tau), \quad z_{1}<z<z_{2} \\
\xi_{\mathrm{III}}=h_{\mathrm{III}}(z) g_{\mathrm{III}}(\tau), \quad z_{2}<z<1 .
\end{gathered}
$$

For region I, substitution of Eq. 15 into Eq. 8 leads to Eq. 18.

$$
\frac{1}{g_{\mathrm{I}}} \frac{\mathrm{d} g_{\mathrm{I}}}{\mathrm{d} \tau}=\frac{1}{h_{\mathrm{I}}}\left(\frac{\mathrm{d}^{2} h_{\mathrm{I}}}{\mathrm{d} z^{2}}+\frac{1}{\mathrm{z}} \frac{\mathrm{d} h_{\mathrm{I}}}{\mathrm{d} z}\right)=-\beta_{\mathrm{I}}^{2}
$$

The variable $\beta_{\mathrm{I}}^{2}$ is a separation constant for region I. We, thus, derive the following ordinary differential equations for $g_{I}$ and $h_{I}$.

$$
\begin{gathered}
\frac{\mathrm{d} g_{\mathrm{I}}}{\mathrm{d} \tau}+\beta_{\mathrm{I}}^{2} g_{\mathrm{I}}=0 \\
\frac{\mathrm{d}^{2} h_{\mathrm{I}}}{\mathrm{d} z^{2}}+\frac{1}{z} \frac{\mathrm{d} h_{\mathrm{I}}}{\mathrm{d} z}+\beta_{\mathrm{I}}^{2} h_{\mathrm{I}}=0
\end{gathered}
$$

Equations (19) and (20) have the following solutions:

$$
\begin{gathered}
g_{\mathrm{I}}=\mathrm{e}^{-\beta_{\mathrm{I}}^{2} \tau} \\
h_{\mathrm{I}}=a_{\mathrm{I}} J_{0}\left(\beta_{\mathrm{I}} z\right)+b_{\mathrm{I}} Y_{0}\left(\beta_{\mathrm{I}} z\right),
\end{gathered}
$$

where $J_{0}$ and $Y_{0}$ are Bessel functions of the first and second kinds, respectively, and $a_{\mathrm{I}}$ and $b_{\mathrm{I}}$ are constants. Without loss of generality, we set the integration constant for $g_{\mathrm{I}}$ to be unity.

Similar analyses can be performed for regions II and III, yielding the following:

$$
\begin{gathered}
g_{\mathrm{II}}=\mathrm{e}^{-\beta_{\mathrm{II}}^{2} \tau} \\
h_{\mathrm{II}}=a_{\mathrm{II}} J_{0}\left(\frac{\phi^{1 / 2}}{\gamma^{1 / 2}} \beta_{\mathrm{II}} z\right)+b_{\mathrm{II}} Y_{0}\left(\frac{\phi^{1 / 2}}{\gamma^{1 / 2}} \beta_{\mathrm{II} \mathrm{z}}\right) \\
g_{\mathrm{III}}=\mathrm{e}^{-\beta_{\mathrm{III}}^{2} \tau} \\
h_{\mathrm{III}}=a_{\mathrm{III}} J_{0}\left(\beta_{\mathrm{III}} z\right)+b_{\mathrm{III}} Y_{0}\left(\beta_{\mathrm{III}} z\right) .
\end{gathered}
$$

It is noted that solutions can be also found for $\beta_{I}=\beta_{\mathrm{II}}=\beta_{\mathrm{III}}=0$, where these solutions turn out to be constant in space and time.

Applying the interface and boundary conditions enables the $\beta_{\mathrm{I}}, \beta_{\mathrm{II}}$, and $\beta_{\mathrm{III}}$ values and all constants, but one to be evaluated. For example, the interface conditions lead to the conclusion that $\beta_{\mathrm{I}}=\beta_{\mathrm{II}}=\beta_{\mathrm{III}}$, so we thus use the symbol $\beta$ without subscripts. It can also be shown that there is an infinite number of solutions, with each solution having its own unique value of $\beta$ (denoted as $\beta_{\mathrm{n}}$ ) and the general solution is a superposition of all possible solutions, as shown below.

$$
\xi(z, \tau)=\sum_{\mathrm{n}=0}^{\infty} a_{\mathrm{n}} \eta_{\mathrm{n}} \mathrm{e}^{-\beta_{\mathrm{n}}^{2} \tau}
$$


The variables in this general solution are as follows:

$$
\begin{aligned}
& \eta_{\mathrm{n}}=J_{0}\left(\beta_{\mathrm{n}} z\right) U\left(z_{1}-z\right)+\left\{G J_{0}\left[\left(\frac{\phi}{\gamma}\right)^{1 / 2} \beta_{\mathrm{n}} z\right]+H Y_{0}\left[\left(\frac{\phi}{\gamma}\right)^{1 / 2} \beta_{\mathrm{n}} z\right]\right\} \\
& \times U\left(z-z_{1}\right) U\left(z_{2}-z\right)+L\left[J_{0}\left(\beta_{\mathrm{n}} z\right)-\frac{J_{1}\left(\beta_{\mathrm{n}}\right)}{Y_{1}\left(\beta_{\mathrm{n}}\right)} Y_{0}\left(\beta_{\mathrm{n}} z\right)\right] U\left(z-z_{2}\right) \\
& G=\left(\frac{J_{0}\left(\beta_{\mathrm{n}} z_{1}\right)}{Y_{0}\left[\left(\frac{\phi}{\gamma}\right)^{1 / 2} \beta_{\mathrm{n}} z_{1}\right]}-\frac{1}{\left(\gamma \phi_{w}\right)^{1 / 2}} \frac{J_{1}\left(\beta_{\mathrm{n}} z_{1}\right)}{Y_{1}\left[\left(\frac{\phi}{\gamma}\right)^{1 / 2} \beta_{\mathrm{n}} z_{1}\right]}\right) \\
& \times\left(\frac{J_{0}\left[\left(\frac{\phi}{\gamma}\right)^{1 / 2} \beta_{\mathrm{n}} z_{1}\right]}{Y_{0}\left[\left(\frac{\phi}{\gamma}\right)^{1 / 2} \beta_{\mathrm{n}} z_{1}\right]}-\frac{J_{1}\left[\left(\frac{\phi}{\gamma}\right)^{1 / 2} \beta_{\mathrm{n}} z_{1}\right]}{Y_{1}\left[\left(\frac{\phi}{\gamma}\right)^{1 / 2} \beta_{\mathrm{n}} z_{1}\right]}\right)^{-1} \\
& H=\frac{J_{0}\left(\beta_{\mathrm{n}} z_{1}\right)}{Y_{0}\left[\left(\frac{\phi}{\gamma}\right)^{1 / 2} \beta_{\mathrm{n}} z_{1}\right]}-G \frac{J_{0}\left[\left(\frac{\phi}{\gamma}\right)^{1 / 2} \beta_{\mathrm{n}} z_{1}\right]}{Y_{0}\left[\left(\frac{\phi}{\gamma}\right)^{1 / 2} \beta_{\mathrm{n}} z_{1}\right]} \\
& L=\frac{G J_{0}\left[\left(\frac{\phi}{\gamma}\right)^{1 / 2} \beta_{\mathrm{n}} \mathrm{z}_{2}\right]+H Y_{0}\left[\left(\frac{\phi}{\gamma}\right)^{1 / 2} \beta_{\mathrm{n}} \mathrm{z}_{2}\right]}{J_{0}\left(\beta_{\mathrm{n}} \mathrm{z}_{2}\right)-\frac{J_{1}\left(\beta_{\mathrm{n}}\right)}{Y_{1}\left(\beta_{\mathrm{n}}\right)} Y_{0}\left(\beta_{\mathrm{n}} \mathrm{z}_{2}\right)} .
\end{aligned}
$$

The variable $U$ is the Heaviside step function such that $U(x)=0$ if $x<0$ and $U(x)=$ 1 if $x>0$. The $\beta_{\mathrm{n}}$ values, which are eigenvalues, are solutions of Eq. 32 .

$$
\frac{J_{1}\left(\beta_{\mathrm{n}} z_{2}\right)-\frac{J_{1}\left(\beta_{\mathrm{n}}\right)}{Y_{1}\left(\beta_{\mathrm{n}}\right)} Y_{1}\left(\beta_{\mathrm{n}} z_{2}\right)}{J_{0}\left(\beta_{\mathrm{n}} z_{2}\right)-\frac{J_{1}\left(\beta_{\mathrm{n}}\right)}{Y_{1}\left(\beta_{\mathrm{n}}\right)} Y_{0}\left(\beta_{\mathrm{n}} z_{2}\right)} \frac{G J_{0}\left[\left(\frac{\varphi}{\gamma}\right)^{1 / 2} \beta_{\mathrm{n}} z_{2}\right]+H Y_{0}\left[\left(\frac{\varphi}{\gamma}\right)^{1 / 2} \beta_{\mathrm{n}} z_{2}\right]}{G J_{1}\left[\left(\frac{\varphi}{\gamma}\right)^{1 / 2} \beta_{\mathrm{n}} z_{2}\right]+H Y_{1}\left[\left(\frac{\varphi}{\gamma}\right)^{1 / 2} \beta_{\mathrm{n}} z_{2}\right]}=(\varphi \gamma)^{1 / 2}
$$

Due to the spatially-discontinuous nature of the properties $\phi$ and $\gamma$, the $\eta_{\mathrm{n}}$ functions are not orthogonal with respect to $z$ and cannot be used directly to calculate values for the constants $a_{\mathrm{n}}$. However, closely related functions can be defined that are orthogonal with respect to $z$ (Tittle 1965; de Monte 2000, 2002) and which enable the constants $a_{\mathrm{n}}$ to be calculated. These functions, denoted as $\delta_{\mathrm{n}}$, are listed below.

$$
\begin{aligned}
\delta_{\mathrm{n}}= & J_{0}\left(\beta_{\mathrm{n}} z\right) U\left(z_{1}-z\right)+\phi^{1 / 2}\left\{G J_{0}\left[\left(\frac{\phi}{\gamma}\right)^{1 / 2} \beta_{\mathrm{n}} z\right]+H Y_{0}\left[\left(\frac{\phi}{\gamma}\right)^{1 / 2} \beta_{\mathrm{n}} z\right]\right\} \\
& \times U\left(z-z_{1}\right) U\left(z_{2}-z\right)+L\left[J_{0}\left(\beta_{\mathrm{n}} z\right)-\frac{J_{1}\left(\beta_{\mathrm{n}} z\right)}{Y_{1}\left(\beta_{\mathrm{n}} z\right)} Y_{0}\left(\beta_{\mathrm{n}} z\right)\right] U\left(z-z_{2}\right)
\end{aligned}
$$

The constants $a_{\mathrm{n}}$ are then determined as shown in Eq. 34 .

$$
a_{\mathrm{n}}=\frac{\int_{0}^{z_{1}} z \eta_{\mathrm{n}} \mathrm{d} z+\phi \int_{z_{1}}^{z_{2}} z \eta_{\mathrm{n}} \mathrm{d} z}{\int_{0}^{z_{1}} z \eta_{\mathrm{n}}^{2} \mathrm{~d} z+\phi \int_{\mathrm{z}_{1}}^{\mathrm{z}_{2}} z \eta_{\mathrm{n}}^{2} \mathrm{~d} z+\int_{\mathrm{z}_{2}}^{1} z \eta_{\mathrm{n}}^{2} \mathrm{~d} z}
$$


Table 3 Representative values of $\beta_{1}, \beta_{2}, a_{1}$, and $a_{2}$ from the exact solution as a function of $\gamma, z_{1}$, and $z_{2}$ with $\phi=0.151$

\begin{tabular}{lllllll}
\hline$z_{1}$ & $z_{2}$ & $\gamma$ & $\beta_{1}$ & $\beta_{2}$ & $a_{1}$ & $a_{2}$ \\
\hline & & & & & & \\
0.1309 & 0.1587 & 0.05 & 3.736 & 5.321 & 0.326 & 0.685 \\
0.1309 & 0.1587 & 0.02 & 3.121 & 4.349 & 0.869 & 0.141 \\
0.1309 & 0.1587 & 0.01 & 2.353 & 4.176 & 0.984 & 0.0200 \\
0.1309 & 0.1587 & 0.005 & 1.704 & 4.125 & 0.996 & 0.00352 \\
0.1309 & 0.1587 & 0.002 & 1.091 & 4.102 & 0.995 & 0.000387 \\
0.1309 & 0.1587 & 0.001 & 0.774 & 4.095 & 0.994 & 0.0000376 \\
0.4075 & 0.4946 & 0.05 & 1.801 & 6.419 & 0.875 & 0.00663 \\
0.4075 & 0.4946 & 0.02 & 1.188 & 6.327 & 0.845 & -0.0000576 \\
0.4075 & 0.4946 & 0.01 & 0.852 & 6.279 & 0.834 & -0.00160 \\
0.4075 & 0.4946 & 0.005 & 0.607 & 6.094 & 0.827 & -0.0139 \\
0.4075 & 0.4946 & 0.002 & 0.386 & 4.150 & 0.824 & -0.0207 \\
0.4075 & 0.4946 & 0.001 & 0.273 & 2.499 & 0.822 & -0.0170 \\
\hline
\end{tabular}

For $n=0$, which corresponds to $\beta_{0}=0$, we obtain the following expression for $a_{0}$.

$$
a_{0}=\frac{z_{1}^{2}+\phi\left(z_{2}^{2}-z_{1}^{2}\right)}{1+(\phi-1)\left(z_{2}^{2}-z_{1}^{2}\right)}
$$

At the center of the porous cup we obtain Eq. 36 if we apply the initial condition $C_{1}=0$.

$$
\frac{C(0, t)}{C_{2}}=1-\frac{z_{1}^{2}+\phi\left(z_{2}^{2}-z_{1}^{2}\right)}{1+(\phi-1)\left(z_{2}^{2}-z_{1}^{2}\right)}-\sum_{\mathrm{n}=1}^{\infty} a_{\mathrm{n}} \mathrm{e}^{-\beta_{\mathrm{n}}^{2} D t / r_{3}^{2}}
$$

It is noted that $C_{\text {ave }}=C_{2}\left(1-a_{0}\right)$ is the average concentration attained everywhere in the system at infinite time. For sufficiently large times, the first term in the summation is dominant, yielding

$$
\frac{C(0, t)}{C_{2}} \approx 1-\frac{z_{1}^{2}+\phi\left(z_{2}^{2}-z_{1}^{2}\right)}{1+(\phi-1)\left(z_{2}^{2}-z_{1}^{2}\right)}-a_{1} \mathrm{e}^{-\beta_{1}^{2} D_{\mathrm{o}} t / r_{3}^{2}} .
$$

Shown in Table 3 are values of $\beta_{1}, \beta_{2}, a_{1}$, and $a_{2}$ for $\phi=0.151$ and various $\gamma, z_{1}$, and $z_{2}$ values from our experiments. These values, and others presented later, were generated using the software package Mathematica Version 5.2 (Wolfram Research, Inc 2005). By considering the $\beta_{1}$ and $\beta_{2}$ values in Table 3 , it is apparent that the first term in the summation becomes dominant at smaller values of $\tau$ as $\gamma$ decreases.

\subsection{An Approximate Solution to the Governing Equations}

We will now develop an approximate analytical solution that is valid for the situation where $\gamma=D_{\mathrm{w}} / D_{\mathrm{o}}<<1$. An advantage of this type of approach is it can provide more physical insight into the controlling factors. For example, a critical factor in the exact analysis turned out to be the eigenvalue $\beta_{1}$. The exact expression that relates $\beta_{1}$ to $\gamma$, i.e., Eq. 32, is complicated and it is difficult to relate $\beta_{1}$ to other variables by using the exact analysis. With the approximate analysis, however, $\beta_{1}$ can be related to other variables in a more transparent 
fashion, which would be difficult to achieve with numerical or exact analytical methods. We note that this constraint on $\gamma$ applies only for specific conditions, as $D_{\mathrm{o}}$ will have to be replaced by a $D_{\text {soil }}$ value for a soil solution that typically will be much smaller in magnitude than $D_{\mathrm{o}}$, thereby resulting in $\gamma$ values that may be of order unity or perhaps larger. In those circumstances, the approximate solution outlined below will not be valid.

We can make progress by noting that in typical cases of interest, i.e., where the wall is thin, characteristic times for diffusion within the porous wall are small relative to characteristic times for diffusion in the liquid on either side of the wall. For example, in regions I, II, and III, we may define the characteristic diffusion times $t_{I}=r_{1}^{2} / D_{\mathrm{o}}, t_{\mathrm{II}}=\phi\left(r_{2}-r_{1}\right)^{2} / D_{\mathrm{W}}$, and $t_{\mathrm{III}}=\left(r_{3}-r_{2}\right)^{2} / D_{\mathrm{O}}$. The estimates using representative parameter values yield $t_{\mathrm{II}} / t_{\mathrm{I}} \approx 0.23$ and $t_{\mathrm{II}} / t_{\mathrm{III}} \approx 0.15$ for the porous cup used in the present experiments. Under these conditions, the concentration profiles within the porous wall will, after a short initial transient period near the beginning of the diffusion history, be essentially quasi-steady such that time derivatives within the wall are negligible in the first approximation. In order to model this situation, we may thus solve the following differential equations:

$$
\begin{gathered}
\frac{\partial \xi_{\mathrm{I}}}{\partial \tau}=\left(\frac{\partial^{2} \xi_{\mathrm{I}}}{\partial z^{2}}+\frac{1}{z} \frac{\partial v_{\mathrm{I}}}{\partial z}\right), \quad 0<z<z_{1} \\
\frac{\partial^{2} \xi_{\mathrm{II}}}{\partial z^{2}}+\frac{1}{z} \frac{\partial \xi_{\mathrm{II}}}{\partial z}=0, \quad z_{1}<z<z_{2} \\
\frac{\partial \xi_{\mathrm{III}}}{\partial \tau}=\left(\frac{\partial^{2} \xi_{\mathrm{III}}}{\partial z^{2}}+\frac{1}{z} \frac{\partial \xi_{\mathrm{III}}}{\partial z}\right), \quad z_{2}<z<1 .
\end{gathered}
$$

We assume product solutions (separation of variables) to derive the following solutions.

$$
\begin{gathered}
\xi_{\mathrm{I}}=\left[c_{1} J_{0}(\beta z)+f_{1} Y_{0}(\beta z)\right] \mathrm{e}^{-\beta^{2} \tau} \\
\xi_{\mathrm{II}}=\left[c_{2} \ln (z)+f_{2}\right] \mathrm{e}^{-\beta^{2} \tau} \\
\theta_{\mathrm{III}}=\left[c_{3} J_{0}(\beta z)+f_{3} Y_{0}(\beta z)\right] e^{-\beta^{2} \tau}
\end{gathered}
$$

Here, $c_{i}$ and $f_{i}$ are constants $(i=1,2,3)$. Implementing the boundary and interface conditions (Eqs. 11-13) enables all constants but one (selected to be $c_{1}$ ) to be evaluated, yielding the following:

$$
\begin{gathered}
\theta_{\mathrm{I}}=c_{1} J_{0}(\beta z) \mathrm{e}^{-\beta^{2} \tau} \\
\xi_{\text {II }}=c_{1}\left[J_{0}\left(\beta z_{1}\right)-\frac{\beta}{\gamma} z_{1} J_{1}\left(\beta z_{1}\right) \ln \left(\frac{z}{z_{1}}\right)\right] \mathrm{e}^{-\beta^{2} \tau} \\
\xi_{\text {III }}=c_{1}\left[\frac{J_{0}\left(\beta z_{1}\right)-\frac{\beta}{\gamma} z_{1} J_{1}\left(\beta z_{1}\right) \ln \left(\frac{z_{2}}{z_{1}}\right)}{J_{0}\left(\beta z_{2}\right)-\frac{J_{1}(\beta)}{Y_{1}(\beta)} Y_{0}\left(\beta z_{2}\right)}\right]\left[J_{0}(\beta z)-\frac{J_{1}(\beta)}{Y_{1}(\beta)} Y_{0}(\beta z)\right] \mathrm{e}^{-\beta^{2} \tau} .
\end{gathered}
$$

We may also derive the following expression that relates $\gamma$ and $\beta$, thus allowing the eigenvalues to be evaluated.

$$
\gamma=\frac{\beta z_{1} J_{1}\left(\beta z_{1}\right) \ln \left(\frac{z_{2}}{z_{1}}\right)}{J_{0}\left(\beta z_{1}\right)-\frac{z_{1}}{z_{2}} J_{1}\left(\beta z_{1}\right) \frac{J_{0}\left(\beta z_{2}\right)-\frac{J_{1}(\beta)}{Y_{1}(\beta)} Y_{0}\left(\beta z_{2}\right)}{J_{1}\left(\beta z_{2}\right)-\frac{J_{1}(\beta)}{Y_{1}(\beta)} Y_{1}\left(\beta z_{2}\right)}}
$$

This equation will have multiple eigenvalues for a given value of $\gamma$. We will focus on the smallest nonzero eigenvalue, i.e., $\beta_{1}$. If we use a Taylor series expansion of Eq. 47 in terms of $\beta_{1}$, we may derive the following expression. 
Table 4 Exact (Eq. 32), approximate (Eq. 47), and asymptotic (Eq. 49) values for $\beta_{1}$ as a function of $z_{1}, z_{2}$, and $\gamma$ for $\phi=0.151$

\begin{tabular}{lllccc}
\hline$z_{1}$ & $z_{2}$ & $\gamma$ & $\begin{array}{c}\text { Exact } \beta_{1} \\
(\text { Eq. 32) }\end{array}$ & $\begin{array}{c}\text { Approximate } \beta_{1} \\
\text { (Eq. 47) }\end{array}$ & $\begin{array}{c}\text { Asymptotic } \beta_{1} \\
\text { (Eq. 49) }\end{array}$ \\
\hline 0.1309 & 0.1587 & 0.05 & 3.736 & 5.352 & 4.894 \\
0.1309 & 0.1587 & 0.02 & 3.121 & 3.154 & 3.302 \\
0.1309 & 0.1587 & 0.01 & 2.353 & 2.378 & 2.402 \\
0.1309 & 0.1587 & 0.005 & 1.704 & 1.721 & 1.726 \\
0.1309 & 0.1587 & 0.002 & 1.091 & 1.102 & 1.103 \\
0.1309 & 0.1587 & 0.001 & 0.774 & 0.782 & 0.782 \\
0.4075 & 0.4946 & 0.05 & 1.801 & 1.818 & 1.831 \\
0.4075 & 0.4946 & 0.02 & 1.188 & 1.196 & 1.199 \\
0.4075 & 0.4946 & 0.01 & 0.852 & 0.858 & 0.857 \\
0.4075 & 0.4946 & 0.005 & 0.607 & 0.611 & 0.611 \\
0.4075 & 0.4946 & 0.002 & 0.386 & 0.388 & 0.388 \\
0.4075 & 0.4946 & 0.001 & 0.273 & 0.275 & 0.275 \\
\hline
\end{tabular}

$$
\gamma=\frac{z_{1}^{2}\left(1-z_{2}^{2}\right) \ln \left(\frac{z_{2}}{z_{1}}\right)}{2\left(1+z_{1}^{2}-z_{2}^{2}\right)} \beta_{1}^{2}+\frac{z_{1}^{4}\left(z_{2}^{2}-1-2 \ln z_{2}\right) \ln \left(\frac{z_{2}}{z_{1}}\right)}{8\left(1+z_{1}^{2}-z_{2}^{2}\right)^{2}} \beta_{1}^{4}+\cdots
$$

Retaining the first two terms and solving for $\beta_{1}^{2}$ yields Eq. 49.

$$
\beta_{1}^{2} \approx \frac{2\left(1-z_{2}^{2}\right)\left(1+z_{1}^{2}-z_{2}^{2}\right)}{z_{1}^{2}\left(z_{2}^{2}-1-2 \ln z_{2}\right)}\left\{\left[1+\frac{2\left(z_{2}^{2}-1-2 \ln z_{2}\right)}{\left(1-z_{2}^{2}\right)^{2} \ln \left(\frac{z_{2}}{z_{1}}\right)} \gamma\right]^{1 / 2}-1\right\}
$$

Expansion of Eq. 49 for $\gamma \ll 1$ shows that to leading order, $\beta_{1}^{2}$ scales linearly with $\gamma$ as $\gamma \rightarrow 0$.

Shown in Table 4 are values of exact (Eq.32), approximate (Eq.47), and asymptotic (Eq. 49) values of $\beta_{1}$ for $\phi=0.151$ and various $z_{1}$ and $z_{2}$ values from our experiments. These calculations are restricted to small values of $\gamma$, which also correspond to our experiments. The exact, approximate, and asymptotic values for $\beta_{1}$ compare reasonably well as $\gamma$ becomes small. These results will be used to relate a solution based on average concentrations in zones I and III (described next) to the solutions that consider the exact solution species profiles in zones I and III.

\subsection{The Electric Circuit Analog Solution}

A simplified model to predict the time-varying concentrations can also be developed by way of using a simple electric circuit analog (ECA). This model is developed in terms of average concentrations in zones I and III. This type of model is useful, because it provides simplified expressions that can be more transparent in terms of the influences of various parameters. In addition, measurements in the inner cup are taken over a finite volume, and as such the measured data are representative of an average concentration rather than the concentration at a specific point. 
In the analysis, the variable $F$ is defined to be the time rate of change of the volume-average concentration in the inner reservoir, $\bar{C}_{\mathrm{I}}$.

$$
F=V_{\mathrm{I}} \frac{\mathrm{d} \bar{C}_{\mathrm{I}}}{\mathrm{d} t}
$$

where $V_{I}$ is the volume of region I. We also define $R$ to be the overall resistance to diffusion into the interior of the porous cup, such that $F$ is related to $R$ as shown in Eq. 51

$$
F=\frac{\bar{C}_{\text {III }}-\bar{C}_{\mathrm{I}}}{R}
$$

where $\bar{C}_{\text {III }}$ is the volume-average concentration in the outer reservoir.

We require that mass is conserved, or

$$
\bar{C}_{\mathrm{I}} V_{\mathrm{I}}+\bar{C}_{\text {III }} V_{\text {III }}=\bar{C}_{\text {ave }} V_{\mathrm{t}},
$$

where $V_{\mathrm{t}}=V_{\mathrm{I}}+V_{\mathrm{III}}$ and $\bar{C}_{\text {ave }}$ is the average nitrate concentration after full equilibration. By combining Eqs. 50-52, the following ordinary differential equation can be developed to predict $\bar{C}_{\mathrm{I}}$ as a function of time

$$
\frac{\mathrm{d} \bar{C}_{\mathrm{I}}}{\mathrm{d} t}+b \bar{C}_{\mathrm{I}}=b \bar{C}_{\text {ave }},
$$

where

$$
b=\frac{V_{\mathrm{t}}}{R V_{\mathrm{I}} V_{\mathrm{III}}} .
$$

For simplicity, Eq. 53 will be solved subject to the initial condition $\bar{C}_{\mathrm{I}}(0)=C_{1}=0$, leading to $\bar{C}_{\text {ave }}=C_{2} /\left(1+V_{\mathrm{I}} / V_{\mathrm{III}}\right)$. The solution to Eq. 53 can then be written as

$$
\bar{C}_{\mathrm{I}}=\bar{C}_{\text {ave }}\left(1-\mathrm{e}^{-\mathrm{bt}}\right) .
$$

The variable $b$ can be determined by fitting Eq. 55 to experimental data, and by knowing $b$, the overall resistance to nitrate diffusion can be computed by rearranging Eq. 54 .

$$
R=\frac{\mathrm{V}_{\mathrm{t}}}{b V_{\mathrm{I}} V_{\mathrm{III}}}
$$

Further analysis is needed, however, to relate the overall resistance $R$ to other parameters, such as the wall diffusivity and the molecular diffusion coefficient.

In order to make progress, we combine Eqs. 50 and 51 to yield the following expression.

$$
R=\frac{\bar{C}_{\mathrm{III}}-\bar{C}_{\mathrm{I}}}{V_{\mathrm{I}} \frac{\mathrm{d} \bar{C}_{\mathrm{I}}}{\mathrm{d} t}}
$$

We also define the following average values of $\xi$ in regions I and III.

$$
\begin{aligned}
\bar{\xi}_{\mathrm{I}} & =\frac{2}{z_{1}^{2}} \int_{0}^{z_{1}} \xi_{\mathrm{I}} z \mathrm{~d} z \\
\bar{\xi}_{\mathrm{III}} & =\frac{2}{1-z_{2}^{2}} \int_{z_{2}}^{1} \xi_{\mathrm{III}} z \mathrm{~d} z,
\end{aligned}
$$

such that

$$
\bar{C}_{\mathrm{I}}=C_{2}+\left(C_{1}-C_{2}\right) \bar{\xi}_{\mathrm{I}}
$$


and

$$
\bar{C}_{\mathrm{III}}=C_{2}+\left(C_{1}-C_{2}\right) \bar{\xi}_{\mathrm{III}}
$$

The expression for $R$ can then be written as

$$
R=\frac{\bar{\xi}_{\mathrm{III}}-\bar{\xi}_{\mathrm{I}}}{\frac{\mathrm{d} \bar{\xi}_{\mathrm{I}}}{\mathrm{d} \tau}} \frac{\mathrm{r}_{3}^{2}}{D_{\mathrm{o}} V_{\mathrm{I}}}
$$

In general, $R$ will depend on time, but considering the solutions developed earlier it is apparent that $R$ will become essentially constant when the dimensionless time $\tau$ is sufficiently large (e.g., $\tau>0.1$ ). Under these conditions, $\xi_{\text {I }}$ and $\xi_{\text {III }}$ will be dominated by the first eigenvalue $\beta_{1}$ and both $\bar{\xi}_{\text {I }}$ and $\bar{\xi}_{\text {III }}$ will vary as $\mathrm{e}^{-\beta_{1}^{2} \tau}$, enabling Eq. 62 to be written as shown in Eq. 63 .

$$
R \approx\left(1-\frac{\bar{\xi}_{\mathrm{III}}}{\bar{\xi}_{\mathrm{I}}}\right) \frac{r_{3}^{2}}{D_{\mathrm{o}} V_{\mathrm{I}} \beta_{1}^{2}}
$$

Equation (63) is valid for any values of $\gamma$ and $\phi$ (as long as $\tau$ is sufficiently large), but for the present analysis, we will restrict ourselves to the situation, where $\gamma \ll 1$ and where the porous wall can be considered to be quasi-steady, which corresponds to the present experiments. We thus employ Eqs. 44 and 46 to evaluate the ratio $\bar{\xi}_{\mathrm{III}} / \bar{\xi}_{\mathrm{I}}$ as well as Eq. 49 to evaluate $\beta_{1}$. Evaluating the expression for $\bar{\xi}_{\mathrm{III}} / \bar{\xi}_{\mathrm{I}}$ then yields

$$
\frac{\bar{\xi}_{\text {III }}}{\bar{\xi}_{\mathrm{I}}} \approx-\frac{z_{1}^{2}}{1-z_{2}^{2}}
$$

such that

$$
R \approx \frac{1+z_{1}^{2}-z_{2}^{2}}{1-z_{2}^{2}} \frac{r_{3}^{2}}{D_{\mathrm{O}} V_{\mathrm{I}} \beta_{1}^{2}}
$$

Substituting Eq. 49 into Eq. 65 and noting that $V_{\mathrm{I}}=\pi r_{1}^{2} L$, where $L$ is the height of the liquid in the assembly, yields

$$
R \approx \frac{1}{2 \pi D_{\mathrm{o}} L} \frac{z_{2}^{2}-1-2 \ln z_{2}}{\left(1-z_{2}^{2}\right)^{2}}\left\{\left[1+\frac{2\left(z_{2}^{2}-1-2 \ln z_{2}\right)}{\left(1-z_{2}^{2}\right)^{2} \ln \left(\frac{z_{2}}{z_{1}}\right)} \gamma\right]^{1 / 2}-1\right\}^{-1}
$$

Equation (66) can be rearranged to solve for $\gamma$

$$
\gamma \approx \frac{\left(1-z_{2}^{2}\right)^{2} \ln \left(\frac{z_{2}}{z_{1}}\right)}{2\left(z_{2}^{2}-1-2 \ln z_{2}\right)}\left\lfloor\left(1+\frac{z_{2}^{2}-1-2 \ln z_{2}}{\left(1-z_{2}^{2}\right)^{2}} \frac{1}{2 \pi D_{\mathrm{o}} L R}\right)^{2}-1\right\rfloor .
$$

Equation (67) relates the overall resistance to mass transfer $R$, which is obtained by fitting the ECA model to the experiments, to the parameter $\gamma$, enabling $\gamma$ to be estimated from experimental data on average concentrations within zone I.

\subsection{Riga and Charpentier Solution}

The analytical solution introduced by Riga and Charpentier (1998) was also used assuming a constant spatial diffusion coefficient across the inside and outside reservoir, and the 
porous wall, where reduced diffusion across the porous cup wall is neglected. The Riga and Charpentier (RC) solution also assumes radial symmetry across the inside cup and the outside reservoir. The initial uniform concentration inside the porous cup $(t=0), C_{\mathrm{I}}=C_{1}$, is brought into instantaneous contact with an infinite and homogeneous cylindrical source with concentration $C_{\mathrm{III}}=C_{2}$. The general solution is given as Eq.3.10 in Sect.3.2 of Crank (1975). With $C_{\mathrm{I}}=C_{1}=0$, the concentration history at the center of the cup can be written as (Riga and Charpentier 1998)

$$
C_{1}(0, t)=C(0, t)=C_{2} \mathrm{e}^{-r_{2}^{2} / 4 D_{\mathrm{E}} t},
$$

where $D_{\mathrm{E}}$ is effective molecular diffusion coefficient for the liquid/porous wall combination and $C(0, t)$ denotes the nitrate concentration as a function of time at the center of the cup, with $0 \leq t \leq \infty$ in the Re-I.

\section{Comparison of Experimental and Analytical Results}

Figure 2 shows time histories of nitrate concentration in the SS cup as determined from the exact solution (Eq. 36), the ECA solution (Eq. 55), and the RC model (Eq. 68), and how these various solutions compare with the experimental data for each of the four outside reservoirs, with $z_{2}$ values ranging from 0.16 (Fig. 2a) to 0.82 (Fig. 2d). The plots in Fig. 2 shows concentration as a function of physical time. For comparison, Fig. 3 shows the dimensionless concentration $C_{\mathrm{I}} / C_{2}$ as a function of the dimensionless time $\tau$, where $C_{\mathrm{I}}$ is the measured concentration or the predicted concentration at the center of the porous cup in Re-I. The first four terms in the infinite series exact solution were used to plot the exact solution, i.e., Eq. 36, with the solutions forced to be zero at $t=0$. The addition of more terms in the series changed the results only negligibly. For the exact solution and the ECA solution, the wall diffusivity $D_{\mathrm{w}}$, and the variable $b$ were treated as fitting parameters, while $D_{\mathrm{E}}$ was treated as a fitting parameter for the RC model. These parameters were evaluated using the MS Excel solver (Wraith and Or 1998), minimizing an objective function consisting of the sum of the squared error between independent measurements and the model output.

The experimental data in Fig. 2 show that as $z_{2}$ decreases, which corresponds to increases in $r_{3}$, the approach to an equilibrium state takes longer, i.e., the equilibration time increases. This is reasonable, since increases in the outer cup radius will cause the overall resistance to mass transfer to increase, which will increase equilibration times.

An examination of Table 5 and the plots in Figs. 2 and 3 show that all of the analytical models fit the data reasonably well (RMSE $=1.467$ and 1.344 and $R^{2}=0.990$ and 0.992 for ECA and Exact models, respectively) for the smallest $z_{2}$ value $\left(z_{2}=0.16\right)$, though the RC model provided the poorest match $\left(\mathrm{RMSE}=2.242\right.$ and $\left.R^{2}=0.977\right)$. This is to be expected, since the RC model assumes the outer cup is infinitely large. Figures $2 \mathrm{~b}-\mathrm{d}$ and $3 \mathrm{~b}-\mathrm{d}$ show that as $z_{2}$ increases, which corresponds to decreases in the outer cup diameter $\left(r_{3}\right)$, the RC model matches the experimental data less accurately. Again, this is to be expected, since the assumption of an infinitely large outer reservoir is violated more strongly as $z_{2}$ increases.

The plots of the exact solution in Figs. 2 and 3 matches the experimental data better than the RC model as presented by RMSE and $R^{2}$ values for all of the $z_{2}$ values considered, which is reasonable, since the exact solution accounts for the porous wall and the finite size of the outer reservoir (Table 5). However, for the largest value of $z_{2}$ considered (i.e., $z_{2}=0.82$ ), appreciable deviations occur at later times, e.g., for times greater than about $20 \mathrm{~h}(\tau \approx 1)$ (Figs. $2 \mathrm{~d}$ and $3 \mathrm{~d}$ ). These deviations are as a result of the fact that the exact solution does not 

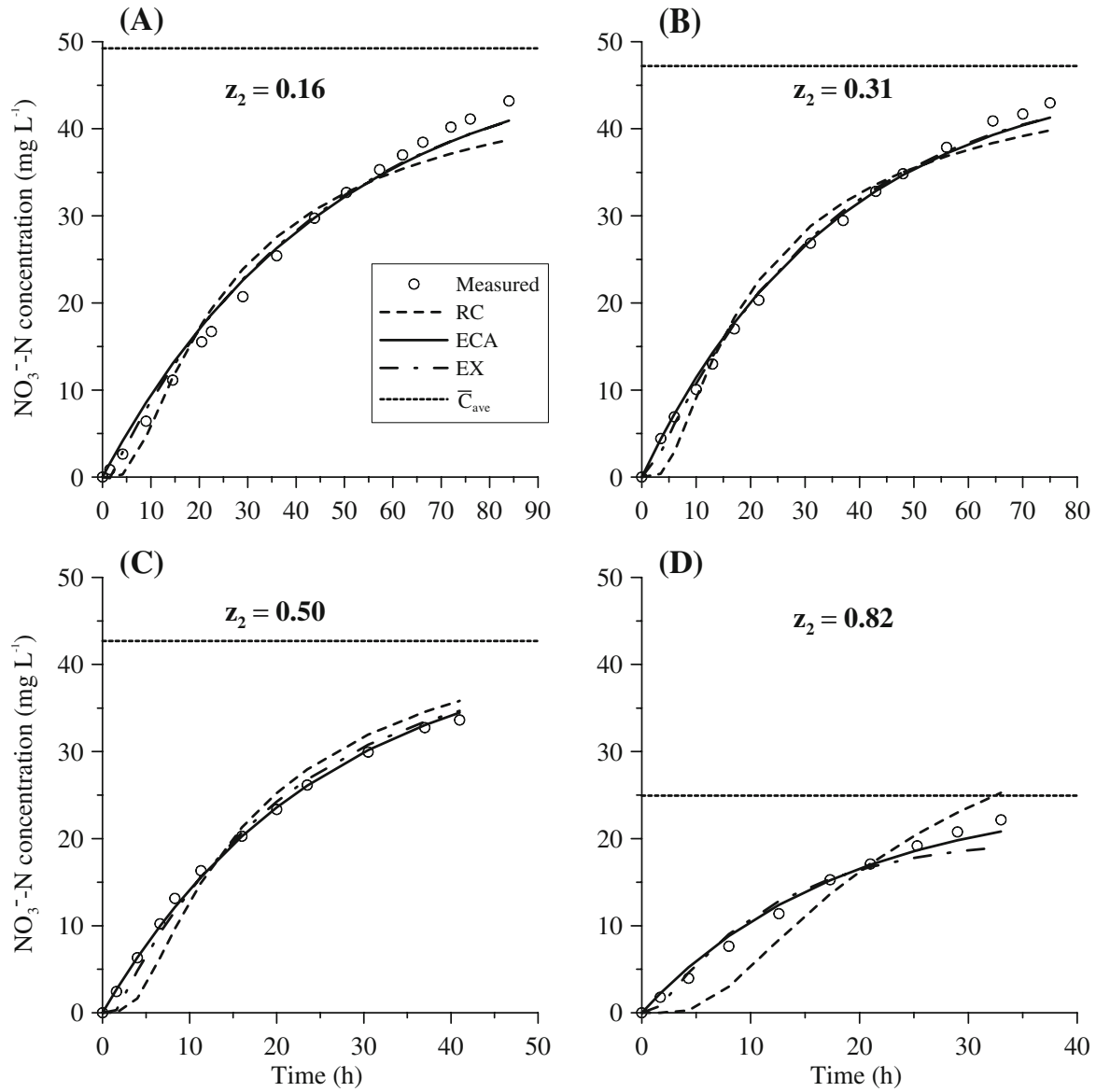

Fig. 2 Comparison of experimental with model concentration data as a function of diffusion time using the RC model, the Electric Circuit Analog (ECA) model and the Exact (EX) model for (a) $z_{2}=0.16,(\mathbf{b}) z_{2}=0.31$, (c) $z_{2}=0.51$, (d) $z_{2}=0.82$. The horizontal lines are the $\bar{C}_{\text {ave }}$ values that applied to the experiments

account for the finite volume of the optical probe, which causes the final average concentration predicted by the exact solution to be different than the actual average concentration in the system. For small $z_{2}$ values (large $r_{3}$ values) the optical probe volume influences the average concentration attained at large times negligibly, which is reflected in the good agreement between the exact solution and the experimental data in Figs. 2 and 3. This can perhaps be seen more clearly by noting that the experiments were performed with the porous cup and its interior initially filled with nitrate-free water, but where the outer cup was loaded with nitrate at the initial concentration $C_{2}$. Conservation of mass arguments yield the following expression for the average concentration $\left(\bar{C}_{\text {ave }}\right)$ of the system.

$$
\bar{C}_{\text {ave }}=\frac{r_{3}^{2}-r_{2}^{2}}{r_{3}^{2}+r_{1}^{2}-r_{2}^{2}+\varphi\left(r_{2}^{2}-r_{1}^{2}\right)-\frac{V_{\mathrm{p}}}{\pi L}}
$$

Here, $V_{\mathrm{p}}$ is the volume of liquid that the optical probe displaces and $L$ is the average depth of the liquid in the inner and outer cups (because the inner cup sat on the bottom of the outer 

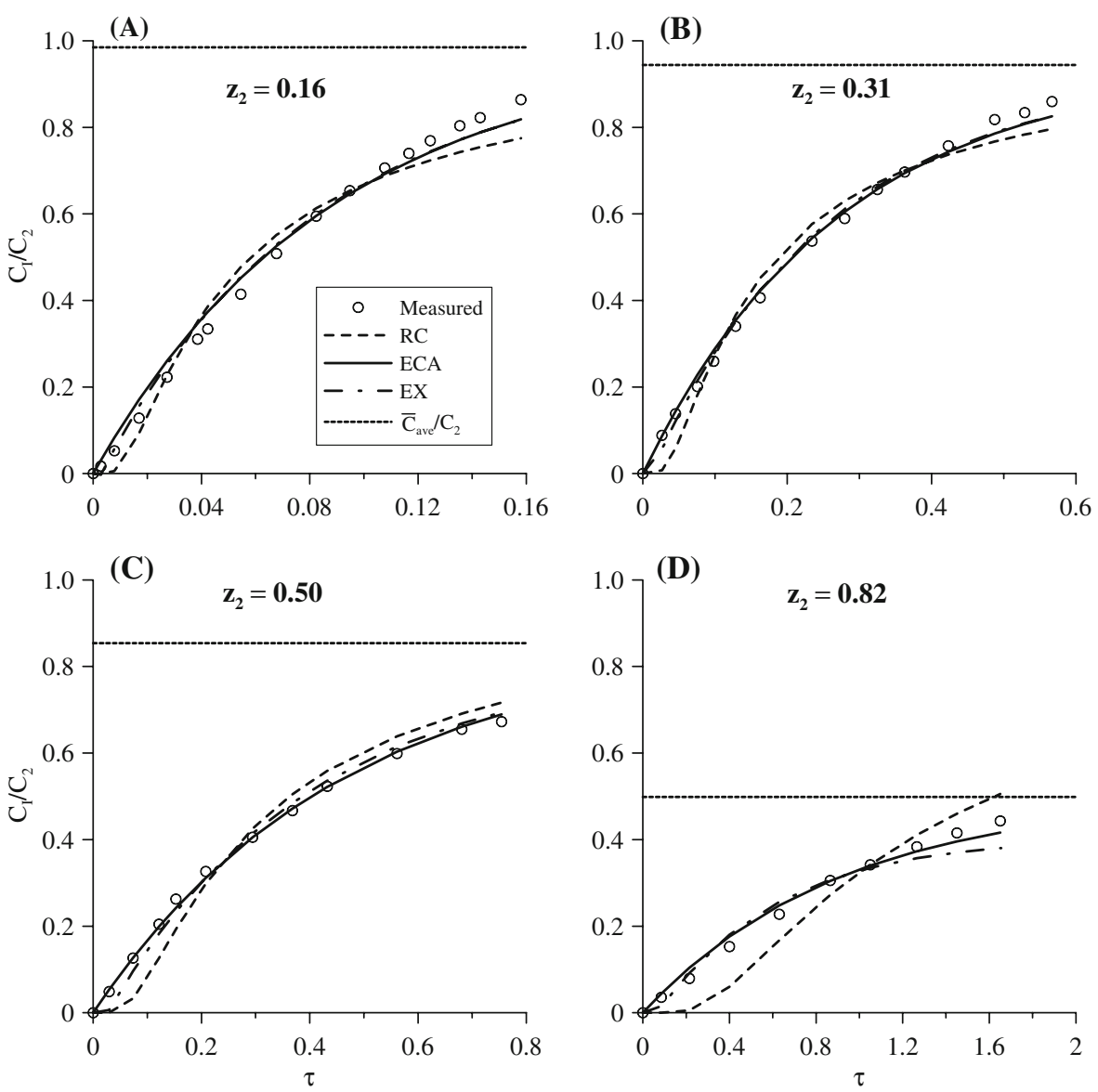

Fig. 3 Comparison of experimental with model solutions, using dimensionless concentration time for porous cup diffusion experiments with the RC model, the Electric Circuit Analog (ECA) model and the Exact (EX) model for (a) $z_{2}=0.16$, (b) $z_{2}=0.31$, (c) $z_{2}=0.50$, (d) $z_{2}=0.82$. The horizontal lines are the ratio $\bar{C}_{\text {ave }} / C_{2}$ for the experiments

cup, the inner-cup depth was slightly smaller than for the outer cup). Examination of Eq. 69 shows that as $r_{3}$ increases, the relative contribution of the term $V_{\mathrm{p}} / \pi L$ decreases such that the optical probe volume decreases, in importance, in terms of determining $\bar{C}_{\text {ave }}$.

The horizontal lines in Fig. 2 are the average concentrations $\bar{C}_{\text {ave }}$ that would exist everywhere in the system at infinite time as predicted by the ECA model (Eq. 55), while the horizontal lines in Fig. 3 are the ratio $\bar{C}_{\text {ave }} / C_{2}$ from the ECA model. The RC solution will, in general, exceed $\bar{C}_{\text {ave }}$ given enough time.

The ECA solution fits the experimental data the best for all of the $z_{2}$ values, considered except smallest $z_{2}$ value (0.16) when RMSE and $R^{2}$ values were examined (Table 5). This is likely due to the fact that the ECA solution explicitly accounts for the probe volume, and also because the ECA solution considers average concentrations while the exact and RC solutions are evaluated at the center of the inner cup. This is important, because the optical probe takes data over a finite region (not at a single point), and the optical data thus represent an average concentration within the probe volume. 


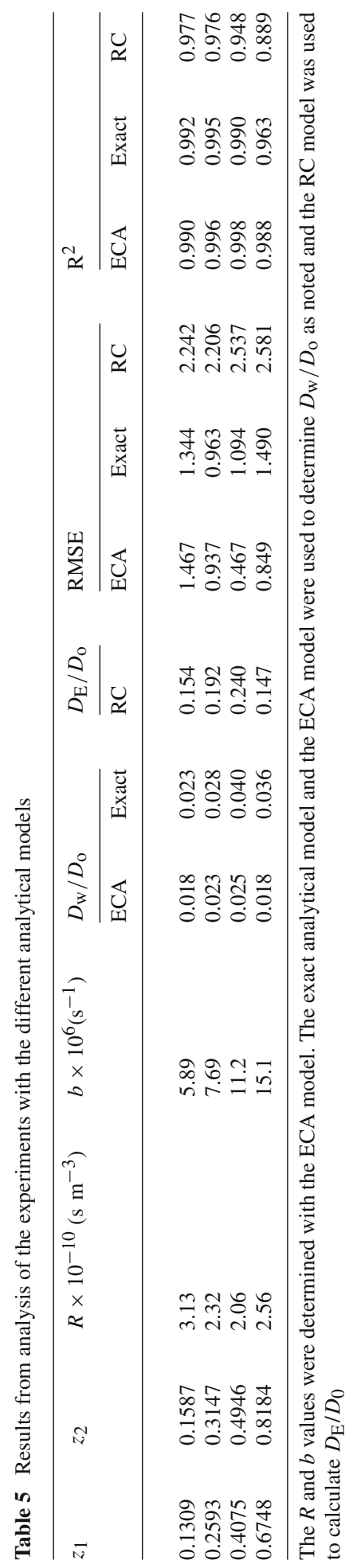


Since the optical probe performs measurements over a finite region, it is worthwhile to calculate average concentrations within the UV probe for comparison with experimental data. The exact solution, Eq. 27, can be averaged over the region covered by the probe, i.e., $0<r<r_{\mathrm{p}}$, where in doing, so we have assumed that the entire probe volume is interrogated by the UV light. This assumption was made because the actual optical path of the UV light within the probe volume was considered to be proprietary information and was not available from the manufacturer. Averaging Eq. 27 over the region $0<r<r_{\mathrm{p}}$ yields Eq. 70 for the average concentration $\bar{C}_{\mathrm{p}}$ within the probe volume

$$
\bar{C}_{\mathrm{p}}=\left(C_{1}-C_{2}\right) \bar{\xi}_{\mathrm{p}}+C_{2},
$$

where $\bar{\xi}_{\text {p }}$ is given by Eq. 71

$$
\bar{\xi}_{\mathrm{p}}=a_{0}+\frac{2}{z_{\mathrm{p}}} \sum_{\mathrm{n}=1}^{\infty} \frac{a_{\mathrm{n}}}{\beta_{\mathrm{n}}} J_{1}\left(\beta_{\mathrm{n}} z_{\mathrm{p}}\right) \mathrm{e}^{-\beta_{\mathrm{n}}^{2} \tau} .
$$

It is noted that $z_{\mathrm{p}}=r_{\mathrm{p}} / r_{3}$.

Equation (70) is plotted in Fig. $4 \mathrm{a}$ for the case $z_{2}=0.31$. For comparison, the centerline concentration $C(0, t)$ from the exact analytical solution (Eq. 36) is also plotted. These data were generated using the value $\gamma=0.028$ (Table 5). The data in Fig. 4a show that $C(0, t)$ lags behind $\bar{C}_{\mathrm{p}}$ slightly, which is reasonable based on physical grounds. In addition, there is an initial time period before the two solutions exhibit appreciable changes. The solution for the average concentration shows appreciable changes before the solution for the centerline concentration.

Figure $4 \mathrm{~b}$ shows predicted species profiles over the entire domain at various times from the exact solution for the case $z_{2}=0.31$. The profiles exhibit gentle gradients in the region, where the optical probe operates, such that the average probe concentration is close to the centerline concentration. The data in Fig. $4 \mathrm{~b}$ show that for an initial period of about $1 \mathrm{~h}$ after the start of an experiment, the nitrate concentration is very small inside the radius corresponding to the probe region $r_{\mathrm{p}}$, even though there are appreciable amounts of nitrate outside of the probe. At later times, concentration gradients in the probe region are very small.

The exact and RC solutions generally predict some time delay before the concentration in the center of the cup (i.e., $z=0$ ) responds. This delay time, which increases in dimensionless time $(\tau)$ as $z_{2}$ increases, but is basically constant in physical time, is generally smaller for the exact solution than for the RC solution (see Figs. 2 and 3). Even though the exact and $\mathrm{RC}$ solutions indicated that appreciable delay times should occur for the larger $z_{2}$ values, these were not observed experimentally. This is consistent with the fact that the measurement volume of the optical probe is not small relative to the size of the SS cup. Consequently, early time concentration changes measured with the UV probe more realistically represent nitrate diffusion across the SS wall into the outer region of the inside of the porous cup thereby causing time delays to be smaller than predicted by the exact and RCA solutions evaluated at $z=0$.

Table 5 also shows results for $R, b, D_{\mathrm{w}} / D_{\mathrm{o}}$, and $D_{\mathrm{E}} / D_{\mathrm{o}}$. These results were obtained by fitting the models to the experimental data in Fig. 2. The exact analytical model and the ECA model were used to determine $D_{\mathrm{w}} / D_{\mathrm{o}}$ (as noted in Table 2) and the RC model was used to calculate $D_{\mathrm{E}} / D_{\mathrm{o}}$. The ECA model was used to calculate values of $D_{\mathrm{w}} / D_{\mathrm{o}}$ by determining $b$ from a fit to the experimental data and then calculating $R$ with Eq. 56. This value of $R$ was then employed in Eq. 67, providing $\gamma=D_{\mathrm{w}} / D_{\mathrm{o}}$.

The $D_{\mathrm{w}} / D_{\mathrm{o}}$ data in Table 5 indicate that the diffusivity of the porous wall was small relative to the molecular diffusivity of nitrate in solution, which is consistent with experimental 
Fig. 4 Calculations from the exact analytical solution for $z_{2}=0.31$ for (a) the centerline and average probe concentrations as a function of time and (b) species profiles calculated for the entire domain. The times listed in (b) are from the beginning of an experiment and the vertical dashed lines show the radius corresponding to the outer edge of the optical probe $\left(r_{\mathrm{p}}\right)$, as well as the inner and outer cup dimensions $\left(r_{1}, r_{2}\right.$, and $\left.r_{3}\right)$

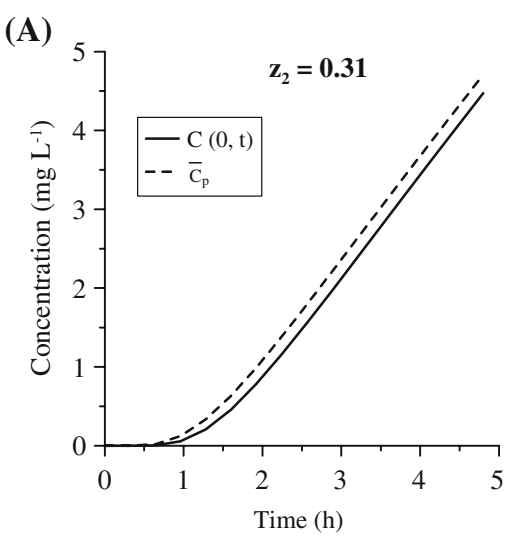

(B)

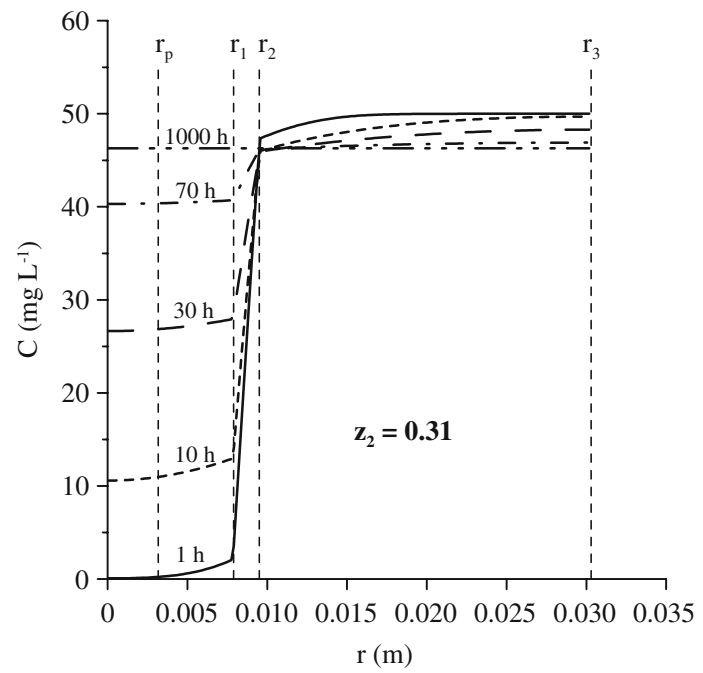

data available in the literature for natural porous media (Grathwohl 1998), as diffusion will be reduced due to the porosity and tortuosity of the porous cup. The $D_{\mathrm{w}} / D_{\mathrm{o}}$ data for the ECA model are similar to but smaller than for the exact model. The smaller $D_{\mathrm{w}} / D_{\mathrm{o}}$ values for the ECA model are likely related to the finite size of the optical probe. The exact solution (Eq. 36) considers only the concentration at $z=0$, while the optical probe measures concentrations across a finite region, leading to faster apparent diffusion rates for Eq.36, since the outer regions of the measurement volume would experience concentration changes before the measurement volume center. As a result, Eq. 36 would lead to larger values of $D_{\mathrm{w}}$ than what would be required for the ECA solution. It is noted that the ratio $D_{\mathrm{w}} / D_{\mathrm{o}}$ should be constant for any value of $z_{1}$ or $z_{2}$. However, uncertainties in the measurements will invariably cause variations in the $D_{\mathrm{w}} / D_{\mathrm{o}}$ values that are determined from the theory.

Overall, the $D_{\mathrm{E}} / D_{\mathrm{o}}$ values are generally several times larger than corresponding $D_{\mathrm{w}} / D_{\mathrm{o}}$ values, but for all cases the ratio $D_{\mathrm{E}} / D_{\mathrm{o}} \ll 1$, as would be expected. This is reasonable, as the RC model employs an effective diffusivity value that accounts for molecular diffusion in the inner (Re-I) and outer (Re-III) liquids as well as diffusion through the porous wall 
(Re-II). We would thus expect that $D_{\mathrm{E}}$ should be larger than $D_{\mathrm{w}}$ but smaller than $D_{\mathrm{o}}$, which is the situation observed here. The large $D_{\mathrm{E}} / D_{\mathrm{o}}$ values as determined from fitting the RC model to the experimental data are likely significantly influenced by the large measurement volume of the UV probe, whereas the RC model provides for a point solution in the center of the porous SS cup $(r=0)$. Moreover, the RC model does not account for the changes in the outer reservoir concentration as controlled by its finite size.

The ECA model performed the best overall in terms of modeling the present experiments, which makes it attractive due to its simplicity and ease of use. However, in order to use the ECA model, we must know the parameter $b$, which can be determined from experiments. In addition, if we consider the structure of the exact analytical solution, the ECA model will perform best at later times when only one eigenvalue dominates the temporal behavior of the exact solution. The exact analytical solution and the ECA solution would likely provide better predictions at early times when several terms in the infinite series would be required for high accuracy. The exact analytical solution can also be used to estimate values of $\gamma=D_{\mathrm{w}} / D_{\mathrm{o}}$, thus enabling wall diffusion coefficients to be evaluated, provided that the assumptions in the model are adequately satisfied, i.e., that diffusion occurs only in the radial direction and that the volume of the optical probe is negligible. In the present experiments, these requirements are most closely satisfied for the smaller $z_{2}$ values investigated.

\section{Conclusions}

We have performed experiments on diffusion of aqueous nitrate ions from an outer cylindrical reservoir through the wall of a cylindrical porous cup and into an aqueous solution inside the porous cup. Nitrate concentrations in the inner cup were monitored as a function of time via UV absorption spectroscopy. By changing the inner radius of the outer reservoir, we were able to investigate the influence of the size of the outer reservoir on temporal species profiles as well as the equilibration time. We also evaluated three different analytical models to predict the nitrate level in the inner cup (Re-I) as a function of time: (i) an exact analytical solution (EX) assuming radial symmetry with a porous wall and a finite size outer reservoir, (ii) an analytical model (RC) that assumes that the outer reservoir is infinitely large and that the porous wall has no influence on diffusion, and (iii) and a model based on an electric circuit analogy (ECA).

The experiments showed that the equilibration time increased as the outer reservoir radius increased. This is a result of the fact that the overall resistance to mass transfer between Region I and III increases as the outer reservoir radius increases. Comparisons of the experimental results with the analytical models also showed that the ECA model generally performed the best, though it is noted that this model is expected to perform adequately only over longer time scales after the beginning of a diffusion experiment. At very early times, the exact analytical solution is expected to provide better predictions of nitrate concentration changes.

It is noted that the exact analytical solution can be used in the case, where soil is present outside the cup, but only if the soil diffusivity $D_{\text {soil }}$ is used to characterize diffusion of the outer zone, instead of the ion diffusivity in water $D_{\mathrm{o}}$. However, it causes the solutions to become more complex from the addition of the additional parameter. The ECA solution would also be valid for soils, though careful calibration of the model would be needed, e.g., by performing experiments under well-controlled laboratory conditions. It is also worthwhile to note that the ECA formulation can be applied to situations with diffusion in more than one space dimension, whereas the EX and RC models are restricted to transient diffusion in only one space dimension. An approach based on the ECA model could thus potentially be 
used to provide predictive capabilities for two- or three-dimensional transport from soil or other media into a porous cup, provided that proper calibrations are performed via careful experimentation.

Acknowledgements This work was supported by the National Science Foundation Biocomplexity program by the funded proposal 0410055 - Development of Multi-Functional Heat Pulse Probe for Ecological and Soil Hydrological Monitoring of Plant Root Zones.

Open Access This article is distributed under the terms of the Creative Commons Attribution Noncommercial License which permits any noncommercial use, distribution, and reproduction in any medium, provided the original author(s) and source are credited.

\section{References}

Crank, J.: The Mathematics of Diffusion . 2nd edn. Clarendon Press, Oxford (1975)

de Monte, F.: Transient heat conduction in one-dimensional composite slab: a 'natural' approach. Int. J. Heat Mass Transf. 43, 3607-3619 (2000) doi:10.1016/S0017-9310(00)00008-9

de Monte, F.: An analytic approach to the unsteady heat conduction processes in one-dimensional composite media. Int. J. Heat Mass Transfer 45, 1333-1343 (2002) doi:10.1016/S0017-9310(01)00226-5

Grathwohl, P.: Diffusion in Natural Porous Media: Contaminant Transport, Sorption/Desorption and Dissolution Kinetics. Kluwer Academic Publishers, Boston (1998)

Moutonnet, P., Pagenel, J.F., Fardeau, J.C.: Simultaneous field measurement of nitrate-nitrogen and matric pressure head. Soil Sci. Soc. Am. J. 57, 1458-1462 (1993)

Poss, R., Noble, A.D., Dunin, F.X., Reyenga, W.: Evaluation of ceramic cup samplers to measure nitrate leaching in the field. Eur. J. Soil Sci. 46, 667-674 (1995) doi:10.1111/j.1365-2389.1995.tb01363.x

Riga, P., Charpentier, S.: Ionic-equilibrium time inside ceramic cups in unsaturated porous media. Soil Sci. Soc. Am. J. 62, 574-579 (1998)

Tittle, C.W.: Boundary value problems in composite media: quasi-orthogonal functions. J. Appl. Phys. 36, 1486-1488 (1965) doi:10.1063/1.1714335

Tuli, A., Wei, J.-B., Shaw, B.D., Hopmans, J.W.: In situ monitoring of soil solution nitrate: proof of concept. Soil Sci. Soc. Am. J. 73, 1-9 (2009) doi:10.2136/sssaj2008.0160

Vanysek, P.: Ionic conductivity and diffusion at infinite dilution. In: D.R. Lide Handbook of Chemistry and Physics, pp. 5-76-5-78. CRC Press/Taylor and Francis, Boca Raton, FL (2008)

Weihermuller, L., Siemens, J., Deurer, M., Knoblauch, S., Rupp, H., Gottlein, A., Putz, I.: In situ soil water extraction: a review. J. Environ. Qual. 36, 1735-1748 (2007) doi:10.2134/jeq2007.0218

Wolfram Research, Inc.: Mathematica, Version 5.2, Champaign, IL (2005)

Wraith, J.M., Or, D.: Nonlinear parameter estimation using spreadsheet software. J. Nat. Resour. Life Sci. Educ. 27, 13-19 (1998) 